\title{
The Temperature Warning System Based on the Renesas MCU
}

\author{
Miaomiao Zhu ${ }^{\mathrm{a}, *}$, Shengrong Gong ${ }^{\mathrm{b}}$, Zhenjiang Qian ${ }^{\mathrm{b}}$, Lifeng Zhang ${ }^{\mathrm{a}}$

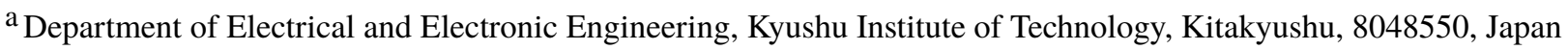 \\ ${ }^{\mathrm{b}}$ School of Computer Science and Engineering, Chang Shu Institute of Technology, Changshu,215500, China \\ *Corresponding Author: zhu.miaomiao234@ mail.kyutech.jp
}

\begin{abstract}
Based on the Renesas 78 KOR series microcontroller, the system uses the temperature sensor to collect real-time temperature, LCD screen to display the date, time and temperature, KEY keyboard to set various parameter values and LED and buzzers test the temperature, which is a visual and audible temperature early warning system. With Renesas energy-saving products $\mathrm{MCU}$ as the core and using the CubeSuite programmed $\mathrm{C}$ language in the special integrated development environment, the product can be widely used in people's daily life and production with good prospects in application and promotion.
\end{abstract}

keywords: Renesas, MCU, LCD, Temperature warning.

\section{Introduction}

In order to respond to "low-carbon society", advocate building a green intelligent society, improve and strengthen the research and development strength of domestic technicians in Microcontroller technology, and actively promote the development and application of domestic energy-saving Microcontroller products. ${ }^{(\mathbb{I})}$ it is of great value and significance to study Renesas Electronics in the world's leading green MCU and its extended functions.

The temperature early warning system can monitor environmental temperature and warn when an exception occurs in early warning function of the system. In many special places and industry it has important use, such as server rooms, book preservation rooms, grain depot and Chinese medicine warehouse, etc., all of which need the specified temperature environmental condition. However, the temperature is the most difficult index to control. In view of this situation, it is vital to design a reliable, convenient and easy temperature warning system to timely warn.With Renesas 16 bits MCU as the core, ${ }^{(2)}$ LM35DZ precision temperature sensors collecting real-time temperature, LCD screens displaying the current real-time temperature, LED lamps displaying temperature warning situation. This paper mainly designs a temperature warning system to achieve LCD and LED display, the hardware, the software and design process are discussed in detailed.

\section{Microcontroller and Design of Hardware}

\subsection{Renesas 78 K0R Series Microcontroller}

At present, with the increasing of the people's environmental protection consciousness, consumers pay more and more attention to power consumption and energy saving of the Microcontroller system. In order to expand its application in energy-efficient appliances and battery power supply system, Renesas electronics add the energy conservation idea into new products and produce the 16-bit low-power consumption flash memory micro controller products 78 KOR series, which are considered to have low-power consumption and high efficiency with a high standard in its field.

With Renesas's excellent technology in the major existing products 78 K0R series in the 8/16-bit MCU market, Renesas $78 \mathrm{~K} 0 \mathrm{R}$ series integrate multiple surrounding functions to make its performance more powerful. It has the following main features: ${ }^{(3)}$

(a) Support the system of smaller size and lower power consumption. The MCU integrate the touch sensor chip capacitor sensor control unit, which means it can use the single chip to process system control and touch control and don't need external touch sensor IC chip anymore and reduce the size of the system. Besides, it can be connected directly with the electrode that is used to implement touch induction. So for each electrode, the requirements to resistors and capacitors are eliminated. Then reducing components and the total cost of the system, improving the reliability, shortening the development time can be realized. 


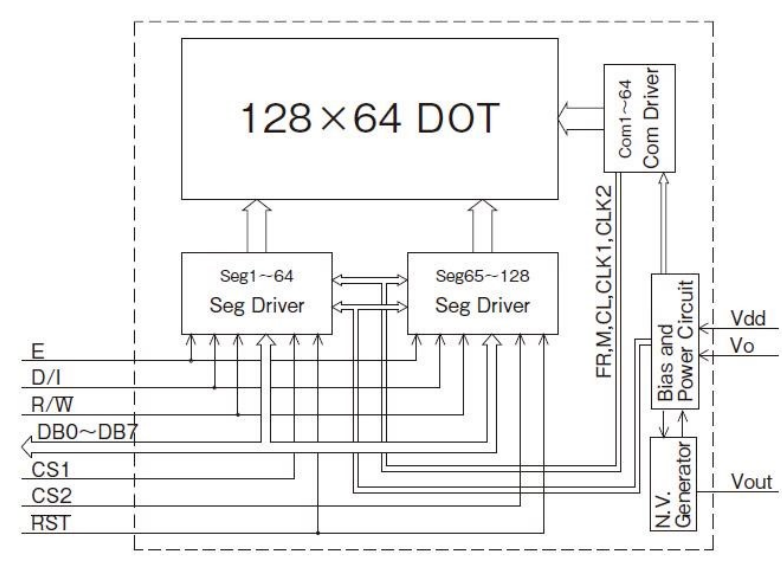

Fig. 1. $128 \times 64$ bitmap graphics LCD foot structure

(b) Reduce the total power consumption of the system. By adding sensor control units, the CPU processing time spending on the touch detection is greatly shortened and the power consumption of the system fall by several times compared with other Renesas similar products in the touch detection of the system. In addition, the sensor control unit can achieve low frequency (4 $\mathrm{MHz}$ or $5 \mathrm{MHz}$ ) operation, so as to further reduce the power consumption.

This design mainly uses Renesas 78 K0R/KG3 series uPD78F1166 chip as the foundation for design and implementation.

\subsection{LM35DZ precision temperature sensor}

This design uses a compact precise temperature LM35DZ. The sensor is connected with the MCU extended interface through the interface, and through the single bus protocol it communicates with the MCU. After the Celsius temperature sensor is converted through A/D it will have parallel threestate output and connect with the microcomputer interface data bus circuit. The hardware connection is very simple and convenient. The sensor's temperature induction ranges from $0{ }^{\circ} \mathrm{C}$ to $+100{ }^{\circ} \mathrm{C}$, and the accuracy is $0.1^{\circ} \mathrm{C}$. This kind of temperature sensitive device can be widely applied to air conditioners, refrigerators, fuzzy control electric rice cookers, electronic water boiler and other precision temperature control occasions.

\subsection{LCD liquid crystal display}

Liquid Crystal Display (LCD) is a kind of low-power consumption of display devices, widely used in portable electronic products. It not only can save electricity but also can display large information and show beautifully and easily. The quality of its display interface has improve compared with the digital interface. In recent years the liquid crystal display technology develops very quickly. LCD display has become the second largest after austenitic stainless display industry. LCD display products have penetrated into every field of people's life.

$\mathrm{LCD}^{(\mathbb{4})}$ is basically consisted by two pieces of photolithography transparent conductive electrode boards, holding a liquid crystal layer, sealing into a partially flat boxes and attaching a Polaroid on the outer surface. It's mainly has three basic parts:

(a) The glass substrates, which is a kind of float glass production with extremely smooth thin surface.

(b) Liquid crystal,Liquid crystal material is the main body of liquid crystal display device.The liquid crystal materials used in the different devices are also different.

(c) Polaroid, also called polarizing piece, is made of plastic membrane materials and coated with optical pressure sensitive adhesive, so it can stick on the surface of the liquid crystal box.

This design mainly adopts the $128 \times 64$ bitmap graphics LCD of MS12864D, which arranges a matrix of lattice points with 64 lines and 128 columns. The size of the point can be designed according to the definition of the display.Lattice points with $8 \times 8$ can display one number so that the output is displayed with 8 lines and 16 columns. This kind of liquid crystal display can be widely used in graphic display output terminals such as games, notebook computers and color television equipment.The leading foot structure as shown in Fig.⿴囗十

\subsection{LED light display}

Eight LED light-emitting diode are neatly arranged in a line on the MCU I/O board and can achieve water light display after being lighted.Each of them correspond to each one of the P5 port on MCU.The LED is connected by the common LED anode, so when the corresponding I/O outputs low electric, the LED lamp is on, and the corresponding I/O outputs high power, the LED is out. Warning level has yellow warning and red warning: when it is the yellow warning, four LED lights flash regularly, when red warning, eight LED lights flash regularly. 


\subsection{KEY keyboard design}

The KEY input keyboard adopts a distribution of 4-row $\times 4$-column matrix. According to its circuit diagram and requirement setting, the keyboard input are 0 9 digits, left and right movement keys and set $1 \sim \operatorname{set} 4$ setting keys. The digital control key realizes the setting of the number, the left and right movement keys realize the movement of the cursor, and the set $1 \sim \operatorname{set} 4$ keys respectively assist the completion of the input setting, start and stop functions of the time and date according to the design requirements.

\subsection{Buzzer warning design}

The design uses the buzzer as the sound warning device. When the warning set value is reached, the buzzer starts to alarm. Compared with the LED, when the yellow warning sounds, the sound of the buzzer is gentle; when it is the red warning, the sound is rapid. The combination of the buzzer and LED display can get a visual and auditory effect of early warning.

\section{Editing development environment}

CubeSuite is a collection of molding development environment offered by Renesas Company and provides unified support for 8 to 32 bit architecture micro controller. ${ }^{(5)}$ When developing the software or program suitable for the MCU, this collection of molding development environment will link all the tools, including compilers, simulators and debuggers to make them complete all the design, code, evaluation and test in same host. According to the type of the software's MCU being developed, the developer, choose different models from the CubeSuite set molding development environment.

The main feature of the CubeSuite ${ }^{(6)}$, (II) is that the software uses the interface editing, refreshing and beautiful. It uses a project tree to meet the requirements of MCU development process. This kind of complex function let developers turn to the expected task by clicking the node. CubeSuite integrates the necessary basic software in Renesas MCU software development in package. When the installation is completed, it can be immediately put into use. CubeSuite reduces the study time spending on the tools. Because in terms of the function, they are the same as all the supported MCU series. The product also has the function of a code generator and support device driver settings through a GUI, which makes it easy to create programs. When a new series of MCU are used for the first time, the setup program of peripherals function setup will not be confused.
At the same time,CubeSuite has "rapid construction" function,every time the source file is changed or saved, CubeSuite can run the construct function in the background, thus greatly shortening the total construction time. Besides, compared with the general editing software, CubeSuite has the great ability of error correction, and it can point out the error and its location exactly during the compilation process. Its basic functions of editing, constructing and debugging will be more powerful, which can provide more simplicity and convenience and achieve more convenient software development.

This design uses the specific Renesas programming debugging tool Minicube2, which is an emulation debugger with programming function to debug during programming. The Minicube2 is suitable for 8-32-bit Renesas microcontrollers and also compatible with non-all-flash Renesas microcontrollers, The compatibility is better than other compiled emulators. The system board needs power supply during the process of developing programming and debugging. During the process of writing and debugging, the system is powered by the Minicube 2 debugger connected to the computer. When the system runs independently after programming and debugging, the integrated power interface and the power supply runs for the system.

\section{Design and programming realization}

\subsection{Hardware design}

The basic idea of this design is collecting real-time temperature through the temperature sensors and sending it to the micro controller. The real-time temperature is real-time displayed on the LCD connected with the micro controller. At the same time, the controller judge the temperature collected. If the real-time temperature is within the demanding temperature range, the LED and the buzzer will not warn. But if it exceeds the demanding temperature range, different LED lights flash to indicate the different levels of warning and the buzzer also provides sound alarm of different frequencies according to the level of warning.

In this design, mainly based on Renesas electronic 16 bits MCU-78 K0R/KG3, through the data line and MCU corresponding extended interface, the I/O board are connected with the temperature sensor, LCD liquid crystal display panel and 8 LED lamps separately. At the same time, the MCU is attached to the computer through the simulation apparatus, one end of which is attached to the MCU and the other end USB interface of which is attached to the computer.

Through the simulation apparatus, the program composed in the computer will be written on the MCU chip and the 


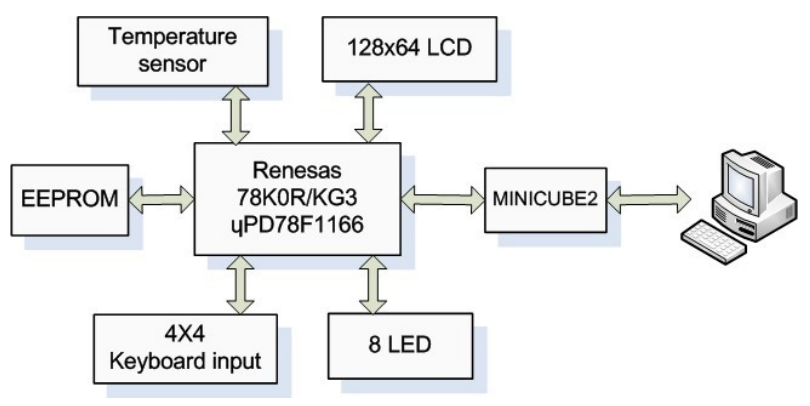

Fig. 2. Hardware modules connected diagram

MCU will be powered constantly. The hardware module connection diagram is shown in Fig. $\square$. The LCD liquid crystal display board shows " $\times \times \times \times($ year $) / \times \times($ month $) / \times \times($ day $)$, $\times \times($ hour $): \times \times($ minute $): \times \times($ second $)$ ", early warning temperature setting value and the current temperature value separately.

\subsection{Software design}

In CubeSuite integrated development environment, choose the MCU of $78 \mathrm{~K} 0 \mathrm{R} / \mathrm{KG} 3$ model and chipset uPD78F1166 (100 pin) to set up a new engineering project. After that, configure and program in accordance with the requirements of the design.

First of all, choose the used port pin in the design tools "port settings" to set and use the pin list to configure multiplexing pin processing. At the same time, confirm the pin layout in the pin configuration chart. According to the corresponding port number of the MCU extended interface and port circuit design, set input and output force for each port pin. Each pin of the temperature sensor access interface P15 is set to be input, carrying on the real-time temperature $\mathrm{A} / \mathrm{D}$ conversion input.

All pins of LCD display interface P8 are set to be output for time and temperature signal. The LCD displays date, time, alarm time, temperature and alarm temperature inline. Eight LED lights in the MCU P5 port pin are set to be output, and the warning level is controlled with the number of the LED, four LED lights flashing for yellow warning and eight for red warning. The buzzer sets the warning frequency for yellow warning and red warning. Set the KEY keyboard input and output pin interface for P7, achieving the setting for date, time and early warning temperature, and the cursor control and screen switch, etc. After the setting, build the terminal, and how each pin of the MCU is set up will be shown in the terminal configuration chart, as shown in Fig. [3].

In the "Timer Settings", open the channel 0, where through internal timer set clock frequency $1000 \mathrm{~ms}$ as a stopwatch unit, and then complete the terminal generation, which will

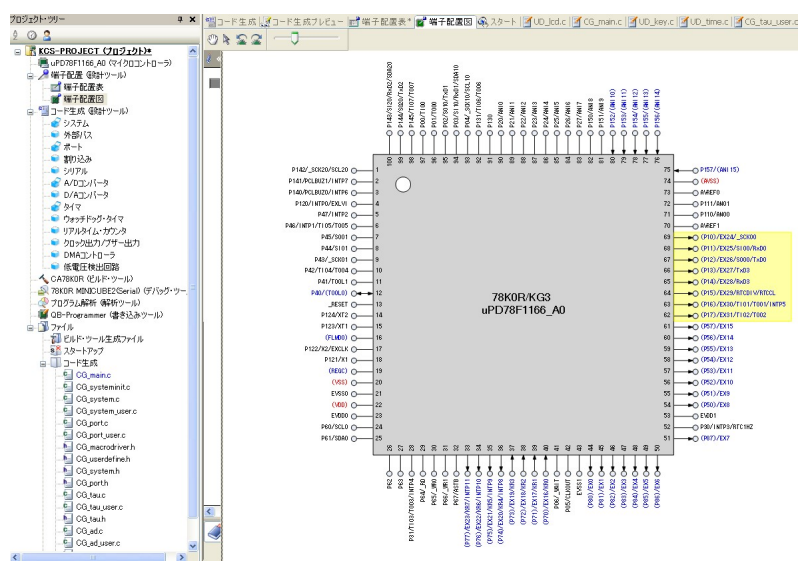

Fig. 3. Terminal configuration

be shown in the terminal configuration chart. In compiling and writing emulator, choose 78 K0R MINICUBE2 (Serial) as a debugging tool. $78 \mathrm{~K} 0 \mathrm{R} / \mathrm{KG} 3$ uses VDD, FLMD0, RESET, TOOL0, TOOL1 and VSS for pins and communicates with the host computer through the chip debugging emulator QB-MINI2 for the chip debugging. This is a flash memory programmer, used especially for the micro controller, and also can be used as the chip debugging emulator. During the development of $78 \mathrm{~K} 0 \mathrm{R}$ application system, it is used for debugging hardware and software. In "Code generator" it is used to write GG_main,C main program and GG_timer,User.C time program, etc. After the completion, the system will compile the project, and at the same time through the Renesas Debug tool-chip debugger write it on the MCU board memory to preserve. Just powered on, it can operate independently.

\subsection{Final result display}

The system designs the final LCD interface style. The LCD interface display information occupies 5 lines of the 8 lines of the LCD screen. The following items are displayed in the 5 lines: the first line is the top blank line; the second line displays the current date, and the display format is Date: $\times \times \times \times($ year $) / \times \times($ month $) / \times \times($ day $)$; the third line shows the time of the current time zone, and the display format is Time: $\times \times($ hour $): \times \times($ minute $): \times \times($ second $)$; the fourth line shows the specific time of the alarm, the display format is Alarm Time: $\times \times($ hour $): \times \times($ minute $): \times \times($ second $)$; the fifth line is used as the interval blank line; the sixth line shows the current real-time temperature value, and its display format is Temp: $\times \times . \times{ }^{\circ} \mathrm{C}$; the seventh line displays the alarm temperature value, and the display format is Alarm Temp: $\times \times . \times{ }^{\circ} \mathrm{C}$; the eighth line is the bottom blank line. The whole LCD terminal display interface as shown in Fig. 田.

The display interface can be set through the KEY keyboard. 


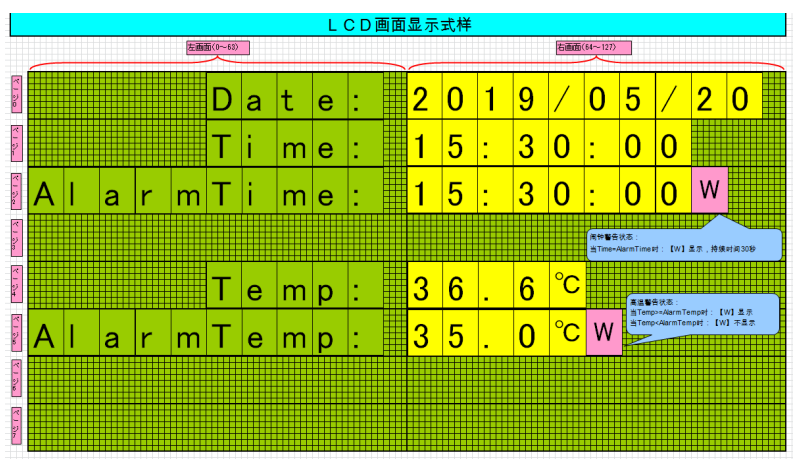

Fig. 4. LCD final display interface

Press the SET button to turn the page into the time zone selection interface which is set to select the time of the three national locations, namely Beijing time, Tokyo time and New York time. After using the keyboard input and controlling the cursor movement to select the time zone, the LCD displays the date and time of the region accordingly.

\subsection{Application}

The temperature warning system can be applied to the server room and monitor its real-time temperature, which is effective. This system can also be used for life feature monitoring, sending and receiving distress signals. The vital sign monitoring module mainly relies on analog-to-digital conversion of various types of sensors to convert various indicators of the human body into visual digital information.

For example, body temperature can be measured by a temperature sensor; heart rate can be measured by a resonant sensor; a pressure sensor can measure blood pressure of a human body. During the monitoring process, if there is an indicator that is lower or higher than the normal value of the human body, an alarm sounds, so that people can timely and intuitively check the status of their own vital signs.

At the same time, the module can also use EEPROM to save the above measured data. The data are written on the on-board EEPROM through the IIC communication protocol. If it is necessary, they can be read out through the on-board LCD screen display, or it is connected with a computer, and the data can be exported to the computer through the RS232 communication protocol. The exported data can be used as reference comparison of future test values. Besides, it can also be regarded as a human black box. When the carrier is in distress it can record his physical condition and provide rescuers with more effective rescue schedules through analysis and processing.

\section{Conclusions}

In the special integrated development environment CubeSuite, based on the Renesas electronic 16 bits MCU-78 K0R /KG3 series $78 \mathrm{f} 1166$ and using the external temperature sensor to collect real-time temperature, this paper uses $\mathrm{C}$ language programming to achieve $128 \times 64$ dot matrix LCD screen display and LED lamp warning function. With reliable performance, Renesas embedded MCU is flexible, convenient, simple, practical and fully functioned. Its idea of protecting the environment and saving energy is being accepted by more and more people so that its practical product will gradually permeate people's daily life and production.

\section{Acknowledgment}

This research was supported by "New generation information technology" Industry-University-Research Innovation Fund Project of Ministry of Education in China under grant No. 2018A01003.

\section{References}

(1) Jing-Yu Xi: "MCU is the mainstay of green environmental protection-Renesas China Forum 2010 focus on hot technology", World Electronic Components, Vol. 1, pp. 70-71, 2011

(2) Zhu Miaomiao, Niu Guofeng, Zhang Mingxin: “The Three-way Interaction Communication Based on Renesas MCU', Journal of ChangShu Institute of Technology,Vol. 4, pp. 89-92, 2016

(3) Xu Junyi, "Renesas Electronics Introduces 8-Bit LowPower Flash Microcontrollers", Industrialization and Informatization Education [J], Vol. 11, pp. 25-26, 2010

(4) Sun Junxi: "LCD driver circuit, driver design and typical applications", Beijing: People's Posts and Telecommunications Press[M], 2009

(5) Zhang Na, Wang Zhidong, Xu Keping: "Application of oscilloscope remote control method in CubeReite integrated development environment CubeSuite+", Electronic Product World, Vol. 11, pp. 57-59, 2014

(6) "Renesas Electronics Introduces Microcontrollers with Ultra Low Power and USB Connectivity", https://www.renesas.com/us/en/about/presscenter/news/2013/news20130416.html, access date: 2017.12 .12

(7) Renesas Technology Corporation: "CubeSuite+ integrated development environment for microcontrollers", Electronic Production,Vol. 7, 2011 